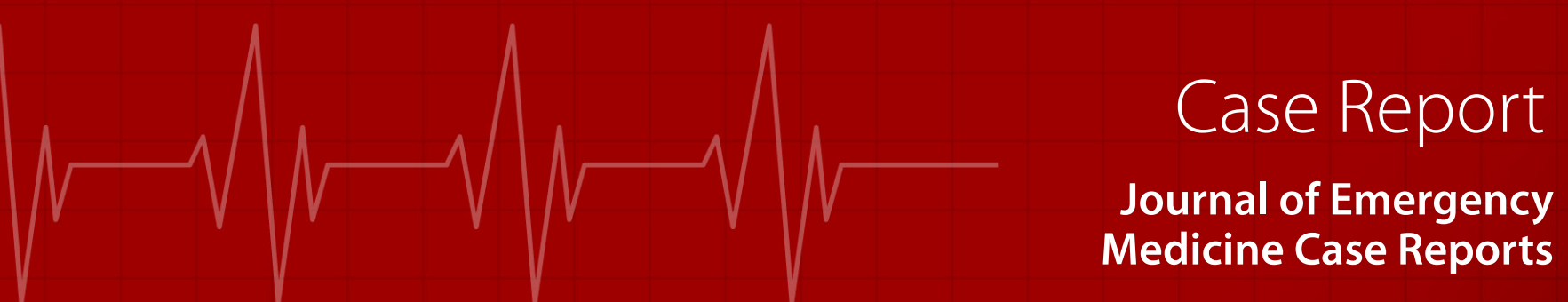

\title{
A Rare Cause of Acute Abdomen in Emergency Room: Isolated Superior Mesenteric Artery Dissection
}

Özlem Güler ${ }^{1}$, Mehmet Buğra Bozan², Seda Nida Karaküçük ${ }^{3}$ Enes Ömer Güngör ${ }^{1}$

${ }^{1}$ Kahramanmaraş Sütçü İmam University Faculty of Medicine, Department of Emergency Medicine ${ }^{2}$ Kahramanmaraş Sütçü İmam University Faculty of Medicine, Department of General Surgery

${ }^{3}$ Kahramanmaraş Sütçü İmam University Faculty of Medicine, Department of Radiology

\section{Abstract}

Introduction: Isolated superior mesenteric artery dissection (ISMAD) has severe and sudden-onset abdominal pain with little physical examination findings. Misdiagnosis of ISMAD is seen commonly in clinical practice, which may lead to severe intestinal ischemic necrosis or even death. Here, we present a patient with abdominal pain and diagnosed as ISMAD.

Case Report: A 46-year-old male was admitted to the emergency department with sudden onset abdominal pain and constipation. He had hypertension and asthma in his history. There was epigastric tenderness in his physical examination. Laboratory tests revealed no pathological findings except high white blood cell count. His complaints did not alleviate despite medical treatment and contrast enhanced abdominal computed tomography was performed. The patient was diagnosed as ISMAD and underwent emergency surgery. The patient developed widespread bowel ischemic necrosis and exitus occurred.

Conclusion: Emergency physicians should be aware of ISMAD and include this in differential diagnoses, especially in patients with acute onset of abdominal pain.

Key Words: Acute abdomen, computerized tomography, isolated superior mesenteric artery dissection

\section{Introduction}

Arterial dissection is a life-threatening condition that can be seen in any artery. Isolated superior mesenteric artery dissection (ISMAD) is a dissecting lesion of the superior mesenteric artery (SMA) and its branches. Compared to other arteries, such as the splenic, hepatic, celiac and gastric arteries, superior mesenteric artery dissection is the most common visceral artery dissection ${ }^{1}$. SMA dissection is usually associated with aortic dissection. With the more common use of multidetector computed tomography, isolated superior mesenteric artery dissections which do not coexist with aortic dissection are reported more frequently today ${ }^{2,3}$.

Isolated superior mesenteric artery dissection has very few clinical findings except severe and sudden onset abdominal pain. The ISMAD clinic may vary from unclear symptoms to fatal SMA rupture ${ }^{2}$. Delays or misdiagnosis of ISMAD can cause severe bowel necrosis and sometimes death $^{4}$.

In this case report, we present a patient with abdominal pain and diagnosed as isolated SMA dissection.

\section{Case Report}

A 46-year-old male was admitted to the emergency department with sudden onset abdominal pain. In his anamnesis he stated that the pain suddenly started in the evening, he had constipation and the pain decreased after enema was administered in another hospital. When the pain increased again later, he applied to our emergency department. $\mathrm{He}$ had hypertension and asthma in his history. His physical examination was normal except epigastric tenderness. Vital findings were: blood pressure $180 / 100 \mathrm{mmHg}$; heart rate $78 /$ $\min$; fever $36.5^{\circ} \mathrm{C}$; $\mathrm{SPO}_{2} 95 \%$; respiration rate $16 / \mathrm{min}$. No pathological findings were observed in the ECG. Laboratory tests revealed no pathological findings other than high white blood cell counts (16.140 U/L). His complaints did not alleviate despite medical treatment and contrast enhanced abdominal computed tomography (CT) was performed. CT showed a double lumen appearance of the dissection starting $3.5 \mathrm{~cm}$ after the superior mesenteric artery origin and continuing through the $4 \mathrm{~cm}$ segment and no luminal filling after dissection (Figure 1,2). The patient was diagnosed as 


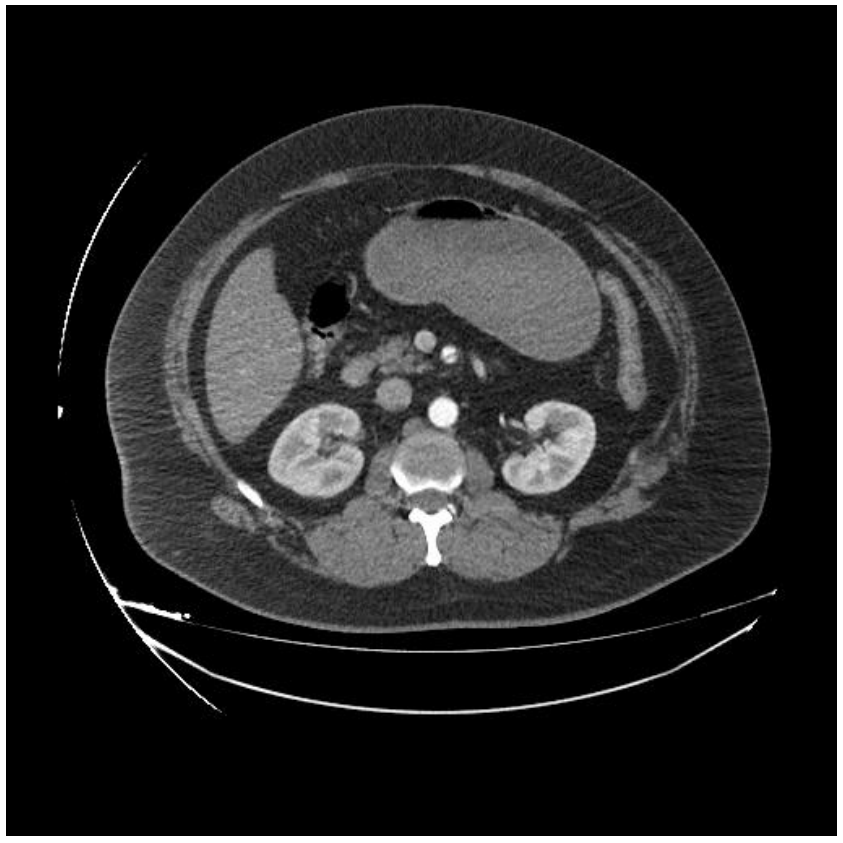

Figure 1: The dissection flap of superior mesenteric artery is seen.

ISMAD and admitted to the general surgery clinic. The patient underwent emergency surgery due to acute abdomen findings during follow-up. Aneurysmatic dilatation was observed at the root of SMA in exploration. The blood supply of right colon and small intestine were disrupted and necrotic areas were observed. A bypass was performed between the SMA and iliac artery. It was observed that the blood supply was returned to $140 \mathrm{~cm}$ area from the ligament of Treitz in intestinal loop. Right hemicolectomy and end-lateral jejunotransvers anastomosis was performed with intestinal resection. Gastrointestinal contents from the abdominal drain were observed during the clinical follow-up and the patient was reoperated with suspicion of anastomotic leakage and the remaining bowel loops were found to be necrotic. The patient developed widespread bowel ischemic necrosis and exitus occurred on the postoperative 2 nd day.

\section{Discussion}

Sudden onset abdominal pain is one of the major causes of emergency admissions even though vascular abdominal pain constitutes only a small part of these cases ${ }^{5}$. Acute mesenteric ischemia (AMI) accounts for $1-2 \%$ of acute abdominal emergencies. Similar to clinical findings in other types of acute mesenteric ischemia, SMA dissection causes severe and sudden onset abdominal pain with very few physical examination findings ${ }^{6}$. Spontaneous ISMAD represents $8 \%$ of all visceral artery dissections and has an overall prevalence of $0.09 \%$ in cadaveric studies $^{7}$. On the other hand increased use of CT revealed that ISMAD was more frequent than previously thought.

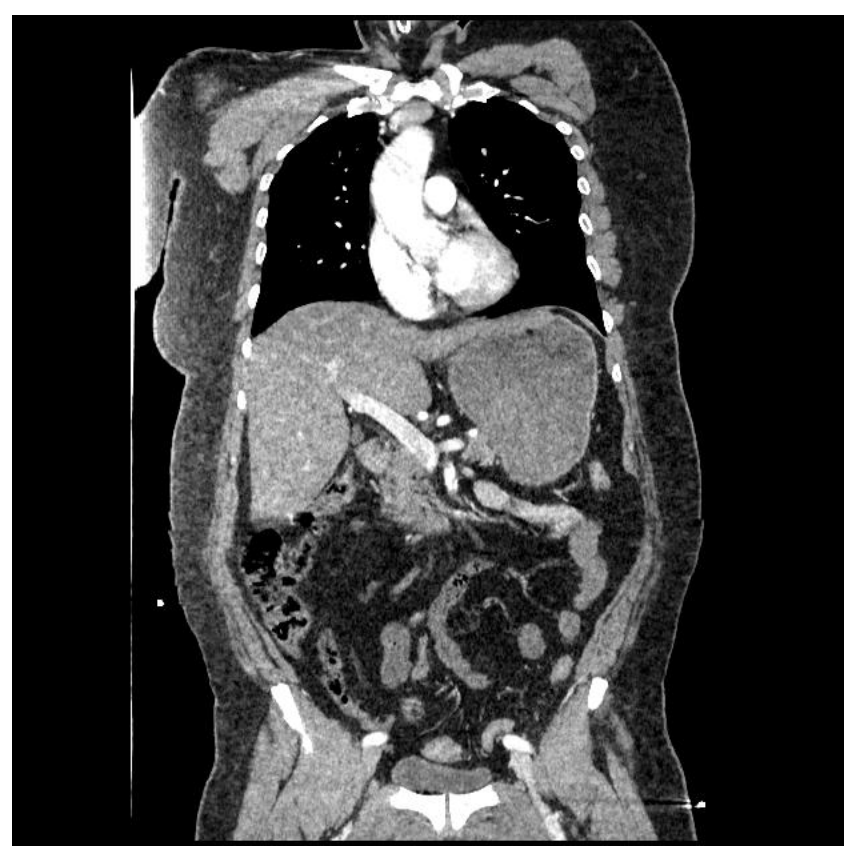

Figure 2: Dissection flap of superior mesenteric artery and disturbed blood flow in the distal part.

The risk factors that cause ISMAD are not fully known. Dou et al. reported that atherosclerosis, smoking and hypertension are the most common risk factors. Connective tissue diseases and genetic susceptibility are also contributing factors to SMA dissection. Strong mechanical stress in the SMA wall is the main factor for SMA dissection ${ }^{6}$. However, no specific underlying cause of SMA dissection has been identified in the majority of reports ${ }^{8}$. Kimura et al reported that isolated SMA dissection was more common in men and hypertension was the most important comorbidity 9 . Similarly our patient was male and had hypertension.

Isolated SMA dissection should be suspected in patients with severe abdominal pain and atherosclerosis. The size of the aneurysm and remaining lumen, the number of collaterals determine the clinical presentation ${ }^{7}$. The main complaint of the patients is sudden onset severe pain. Acute epigastric pain, nausea, vomiting, melena, and abdominal distention are other common clinical findings ${ }^{5}$. Occasionally, patients may be asymptomatic. Rupture of the artery may rarely cause hemorrhagic shock in patients ${ }^{8}$. Our patient presented with acute onset epigastric pain, abdominal distention and constipation.

Direct radiographs or blood tests have no role in the diagnosis of SMA dissection ${ }^{1}$. In this case laboratory findings of the patient were normal except white blood cell count. Symptoms didn't relieve with medical treatment so a contrast enhanced CT angiography was performed in suspect of gastric perforation. CT showed isolated dissection of the SMA. CT angiography can show false lumens and intimal flaps and is helpful in diagnosis. The size of the lesion and bowel infarction can also be detected by CT angiography ${ }^{6}$. Today catheter angiography remains the gold standard diag- 
nostic test for ISMAD. On the other hand, this test should be performed only in patients who require surgical or endovascular treatment and whose condition worsens 9 .

The treatment options for ISMAD include expectant management, anticoagulation, open surgery, and endovascular intervention; however, the optimal initial treatment remains controversial $^{3}$. It was reported that conservative treatment in ISMAD without arterial rupture or bowel necrosis should be first line therapy. Conservative management includes anti-platelet therapy, optimizing blood pressure control, anticoagulation, and bowel rest ${ }^{10}$. Since ISMAD causes low morbidity and mortality medical treatment is advocated. However, results of medical treatment have been reported in series with small number of patients and small dissection sizes ${ }^{7}$.

Emergency revascularization should be applied to patients who are hemodynamically unstable and have radiological progression findings such as formation of thrombus, narrowing or saccular aneurysm formation. Our patient had signs of bowel ischemia so he underwent urgent revascularization and died from total ischemic necrosis. Since SMA dissection is a rare clinical entity, its management, diagnostic methods and outcomes have not been determined in detail yet $^{1}$. Clinical experiences and outcomes related to ISMAD management mainly consist of case series in the literature.

\section{Conclusion}

Isolated SMA dissection is rare and important differential diagnosis of acute abdomen which may result in a life threatening condition such as mesenteric ischemia or aneurysmal SMA rupture. Emergency physicians should be aware of ISMAD and include this in differential diagnoses, especially in patients with acute onset of abdominal pain. Timely and accurate diagnosis is lifesaving.

Conflict of interest: The author have no conficts of interest to declare.

\section{Financial support: None}

\section{References}

1. Ullah W, Mukhtar M, Mohammad AH, Rashid MU, Ahmad A, Hurairah A, et al. Diagnosis and Management of Isolated Superior Mesenteric Artery Dissection: A Systematic Review and Meta-Analysis. Korean Circ J 2019;49:400-8.

2. Daoud $H$, Abugroun $A$, Subahi $A$, Khalaf $H$. Isolated Superior Mesenteric Artery Dissection: A Case Report and Literature Review. Gastroenterol Res 2018;11:374-8.

3. Yan W, Huang R, Shi Q, Shan H, Zhu Y, Cheng G, et al. Multidetector computed tomography in the diagnosis of spontaneous isolated superior mesenteric artery dissection: changes in diameter on nonenhanced scan and stent treatment follow-up. J Int Med Res 2019;47:6139-48.

4. Kim HK, Jung HK, Cho J, Lee JM, Huh S. Clinical and radiologic course of symptomatic spontaneous isolated dissection of the superior mesenteric artery treated with conservative management. J Vasc Surg 2014; 59:46572.

5. Salt Ö, Sayhan MB, Duyar E. Isolated superior mesenteric artery dissection in the emergency department: A rare cause of abdominal pain. Am J Emerg Med 2018;36: 2341.

6. Dou L, Tang H, Zheng P, Wang C, Li D, Yang J. Isolated superior mesenteric artery dissection: CTA features and clinical relevance. Abdom Radiol (NY). 2019 Aug 10. doi: 10.1007/ s00261-019-02171-4.

7. Patelis N, Doukas P, Dodos I, Karampelas T, Kanellopoulos I, Kyriakopoulou K, et al. Endovascular Repair of a Complex Isolated Dissecting Aneurysm of the Superior Mesenteric Artery. EJVES Short Rep 2019; 44: 5-8.

8. Huang CY, Sun JT, Lien WC. Early Detection of Superior Mesenteric Artery Dissection by Ultrasound: Two Case Reports. J Med Ultrasound 2019;27: 47-9.

9. Kimura Y, Kato T, Inoko M. Outcomes of Treatment Strategies for Isolated Spontaneous Dissection of the Superior Mesenteric Artery: A Systematic Review. Ann Vasc Surg 2018;47:284-90.

10. Qiu C, He Y, Li D, Shang T, Wang X, Wu Z, et al. Mid-Term Results of Endovascular Treatment for Spontaneous Isolated Dissection of the Superior Mesenteric Artery. Eur J Vasc Endovasc Surg 2019;58:88-95. 
